\title{
Effect of moderate to severe atopic dermatitis on the prognosis of egg allergy resolution
}

\author{
Seong Heon Kim, ${ }^{1}$ Hye Won Yoo, ${ }^{2}$ Yoon Hee $\mathrm{Jo}^{2}{ }^{2}$ Young Mi Kim, ${ }^{2}$ Hye-Young $\mathrm{Kim}^{2}$
}

\section{Abstract}

Background: Few studies have examined the effect of atopic dermatitis (AD) on the resolution of food allergies in Asia, and the predictors of egg allergy resolution are not yet well defined.

Objective: We evaluated whether AD severity could predict the resolution of egg allergy.

Methods: This retrospective cohort study included infants under 24 months of age diagnosed with IgE-mediated egg white allergy. We included subjects who completed a 60-month follow-up. Open oral food challenges (OFCs) and serologic tests were performed at the time of initial diagnosis and at $36 \pm 3$ and $60 \pm 3$ months.

Results: We analyzed 68 patients (39 boys and 29 girls). OFCs were performed in $88.2 \%$ of the patients. The egg allergy remission rates were $23.5 \%$ and $47.1 \%$ by 3 and by 5 years of age, respectively. Persistent egg allergy was significantly associated with moderate to severe $\mathrm{AD}$ and house dust mite sensitization. Kaplan-Meier curve analysis revealed that patients with moderate to severe $\mathrm{AD}$ had higher persistent egg allergy rates than patients with no and mild $\mathrm{AD}$ $(p=0.012)$. Multivariable analysis identified moderate to severe AD as strongly associated with persistent egg allergy $(p=0.001)$.

Conclusion: In this study, $47.1 \%$ of infants had resolved egg white allergies at 60 months. Moderate to severe AD may be a practical and important prognostic factor for persistent egg allergy in clinical settings.

Key words: Egg allergy, atopic dermatitis, food allergy, prognosis, child

\section{Citation:}

Kim, S. H., Yoo, H. W., Jo, Y. H., Kim, Y. M., Kim, H. Y. (2023) Effect of moderate to severe atopic dermatitis on the prognosis of egg allergy resolution. Asian Pac J Allergy Immunol, 41(1), 30-36. https://doi.org/10.12932/ap-220719-0604

\footnotetext{
Affiliations:

${ }^{1}$ Department of Pediatrics, Research Institute for Convergence of Biomedical Science and Technology, Pusan National University Children's Hospital, Yangsan, Republic of Korea

${ }^{2}$ Department of Pediatrics, Medical Research Institute, Pusan National University School of Medicine,

Pusan National University Hospital, Busan, Republic of Korea

Corresponding author:

Hye-Young Kim

Department of Pediatrics

Pusan National University Hospital, 179 Gudeok-ro, Seo-gu,

Busan 602-739, Republic of Korea

E-mail: pearlhy@naver.com
}

\section{Introduction}

Evaluation and prediction of food allergy resolution are important. Prognostic factors should also be investigated to understand the natural course and management of food allergies in patients. Moreover, due to differences in race, food allergy prevalence, and food intake culture, more studies on the natural course of food allergies in Asian countries, including Korea, are required.

Atopic dermatitis (AD) is a common skin disease characterized by inflammatory, chronically relapsing, and pruritic eczematous flares and marked by skin barrier dysfunction and impaired quality of life. ${ }^{1} \mathrm{AD}$ is the first manifestation of an atopic march which begins in early infancy. Recently, the hypothesis that impaired skin barrier in $\mathrm{AD}$ initiates progression to epicutaneous sensitization, food allergy, and respiratory allergy in later life has become an important topic in the study of allergies. Recent cohort studies showed an increased risk of food allergy and allergic airway disease in late childhood in patients with early-onset persistent $\mathrm{AD} .^{2,3}$ Strong evidence indicates a link between 
early-onset $\mathrm{AD}$ and the development of other allergic diseases, especially food allergy. ${ }^{1,4}$ Approximately $40 \%$ of $\mathrm{AD}$ patients have a food allergy, the prevalence of which is higher in children with severe AD. ${ }^{1,5,6}$ Previous studies on the association between $\mathrm{AD}$ and food allergy focused on the development of food allergy in $\mathrm{AD}$ patients. Some examined the effects of $\mathrm{AD}$ on the natural course of food allergy, mostly in western countries. ${ }^{7,8}$

Therefore, we hypothesized that the skin barrier dysfunction in $\mathrm{AD}$ is a predictor of poor prognosis of food allergy. We focused on patients with egg allergy, one of the most common childhood food allergies, and assessed whether $\mathrm{AD}$ severity could predict the resolution of egg allergy in Korean children.

\section{Methods}

This retrospective cohort study included infants under 24 months of age diagnosed with immunoglobulin $\mathrm{E}$ (IgE)-mediated egg white allergy between January 2011 and December 2013 at Pusan National University Hospital, Busan, Republic of Korea. The eligibility criteria were: (1) diagnosed egg allergy defined by the presence of a positive oral food challenge test (OFC) with egg white or anaphylaxis or repeated episodes of clinical reactions after the ingestion of egg white within 3 months. Patients who could not be administered egg white because of their inherent antigen-specific IgE levels were excluded. (2) Complete follow-up to 60 months of age, and (3) both OFC and serologic tests performed at initial diagnosis and $36 \pm 3$ and $60 \pm 3$ months. This study protocol was approved by the Institutional Review Board of Pusan National University Hospital (PNUHIRB 1801-028-063).

Open OFC was performed initially and at $36 \pm 3$ and $60 \pm 3$ months under the supervision of allergists according to Korean guidelines. ${ }^{9,10}$ Briefly, the patients were challenged with boiled egg white at a total dose of $0.15-0.3 \mathrm{~g}$ protein per kilogram body weight. The total challenging dose did not exceed $3 \mathrm{~g}$ of protein. Over 90 minutes, the patients received egg white in increments of $1,4,10,20,20,20$, and $25 \%$ of the total amount every 15 minutes. The tests were considered positive when the attending pediatric allergist confirmed the appearance of one or more of the following objective symptoms within 2 hours of the last challenge dose; urticaria, angioedema, cough, rhinorrhea, wheezing, stridor, breathing difficulty, vomiting, or low blood pressure. ${ }^{9,11,12}$

Sera from all 68 patients were obtained at the time of the initial visit and at $36 \pm 3$ and $60 \pm 3$ months. Levels of specific IgE (sIgE) antibodies against the allergens (egg white, house dust mite [HDM] Dermatophagoides pteronyssinus [DP] and $D$. farina $[\mathrm{DF}]$ ) were measured by ImmunoCAP (Thermo Fisher Scientific Inc., Waltham, MA, USA). Sensitization was defined as sIgE levels of $0.35 \mathrm{kU} / \mathrm{L}$ or greater. $\operatorname{sIgE}$ antibody levels above $100 \mathrm{kU} / \mathrm{L}$ were assigned a value of $101 \mathrm{kU} / \mathrm{L}$ for analysis.

AD was diagnosed based on the criteria proposed by Hanifin and Rajka. ${ }^{13}$ AD severity was classified using the Scoring Atopic Dermatitis system (SCORAD) as mild $(<15)$, moderate (15-40), or severe ( $>40)$ depending on the area, intensity, and subjective symptoms.
Statistical analysis was performed using IBM SPSS Statistics for Windows, version 20.0 (IBM Corp., Armonk, NY, USA). Data for continuous variables were shown as median and interquartile ranges. Fisher's exact test, Mann-Whitney $U$ test, or one-way analysis of variance (ANOVA) with post hoc comparison tests were used for intergroup comparisons. Cumulative survival curves were estimated by the Kaplan-Meier method and relationships between the cumulative probability of egg tolerance and the prognostic factors were analyzed using log-rank tests. The influence of prognostic factors on egg allergy resolution was evaluated by univariable Cox regression analyses. The relative importance of multiple prognostic factors on egg allergy resolution was analyzed using multivariable analysis in conjunction with the Cox proportional regression model. Variables with $p$-values $<0.2$ in univariable analysis were included in the multivariable analysis. Candidate variables for adjustment included sex, age at diagnosis ( $<1$ or $\geq 1$ year), vaginal delivery (yes or no), exclusive breastfeeding under 6 months (yes or no), anaphylaxis (yes or no), concomitant allergic diseases (presence or absence), family history of allergic diseases (presence or absence), AD severity (none, mild, or moderate to severe), HDM sensitization (negative or positive), total $\operatorname{IgE}$ ( $<200$ or $\geq 200 \mathrm{kU} / \mathrm{L})$, eosinophils $(<4$ or $\geq 4 \%$ ), baseline egg white sIgE at diagnosis $(<20$ or $\geq 20 \mathrm{kU} / \mathrm{L}) . P<0.05$ was considered statistically significant.

\section{Results}

The medical records of 165 patients with IgE-mediated egg white allergy were longitudinally reviewed. Of the patients, 92 received regular follow-up by pediatric allergists. We analyzed 68 patients (39 boys and 29 girls) who both completed the follow-up and underwent regular serologic testing to assess their current status up to 60 months. Sixty participants $(88.2 \%)$ underwent OFC, 8 (11.8\%) of whom had a history of egg allergy (Figure 1).

165 Patients with IgE mediated egg white allergy

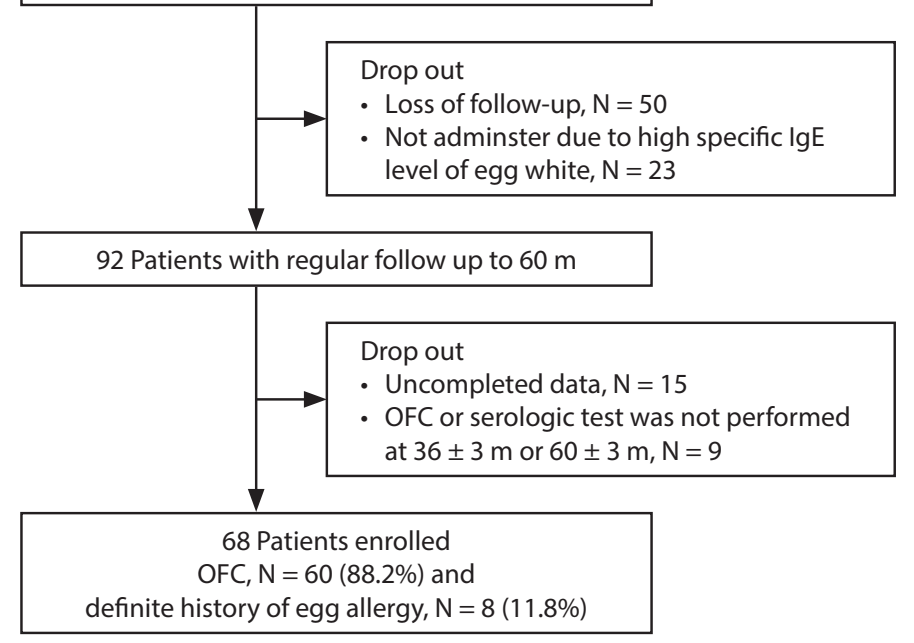

Figure 1. Flow chart showing the inclusion and exclusion of the study participants

m, months; OFC, oral food challenge 
Table 1. Demographic data of children with immunoglobulin E (IgE)-mediated egg white allergy $(\mathrm{N}=68)$

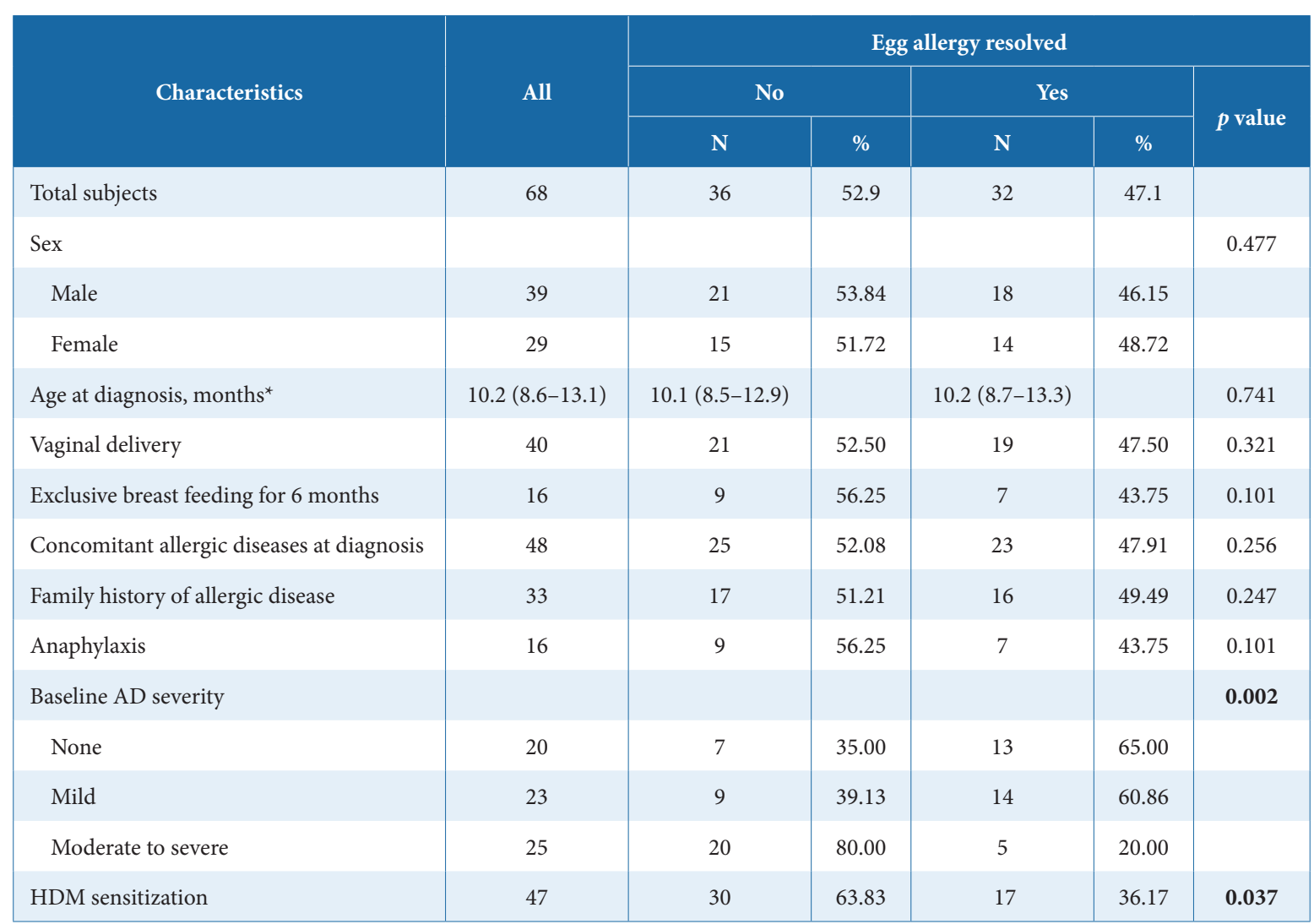

Abbreviations: AD: atopic dermatitis, HDM: house dust mite ${ }^{*}$ median (interquartile range)

The demographic data of these patients are shown in Table 1. The median age of the study subjects at diagnosis was 10.2 years (range 7-16) and anaphylaxis was present in $16(23.5 \%)$ patients. At diagnosis, 20, 23, and 25 patients had no, mild, and moderate to severe AD. The baseline median egg white sIgE level was 19.1 (interquartile range [IQR] 6.7-35.7) kU/ L. Forty-seven (69.1\%) patients were sensitized to HDM. Persistent egg allergy was significantly associated with moderate to severe $\mathrm{AD}$ and $\mathrm{HDM}$ sensitization ( $p=0.002$ and 0.037 , respectively). The median egg white-sIgE levels at 36 and 60 months of age were higher in the persistent egg allergy group than in those in the tolerance group ( $p=0.015$ and 0.010 , respectively) (Figure 2).

Figure 3a shows the Kaplan-Meier curves for egg allergy resolution. The remission rates of the study population with egg allergy were 23.5 and $47.1 \%$ at 36 and 60 months, respectively. Figure $\mathbf{3 b}$ shows that patients with moderate to severe $\mathrm{AD}$ had higher persistent egg allergy rates than those in patients with no and mild $\mathrm{AD}(p=0.008)$. The remission rates of egg allergy at $36 \pm 3$ and $60 \pm 3$ months in the non-AD group were 35 and $65 \%$, respectively; 30 and $56.5 \%$ in the mild $\mathrm{AD}$ group, respectively; and 8 and $24 \%$ in the moderate to severe $\mathrm{AD}$ group, respectively. The median [IQR] egg white-sIgE levels at the time of diagnosis in the no, mild and moderate to severe AD groups were 17.8 [5.4-30.5], 18.9 [7.9-30.3], and 20.7 [9.7-35.7] $\mathrm{kU} / \mathrm{L}$, respectively. No significant differences were found between the three groups at the time of egg allergy diagnosis.

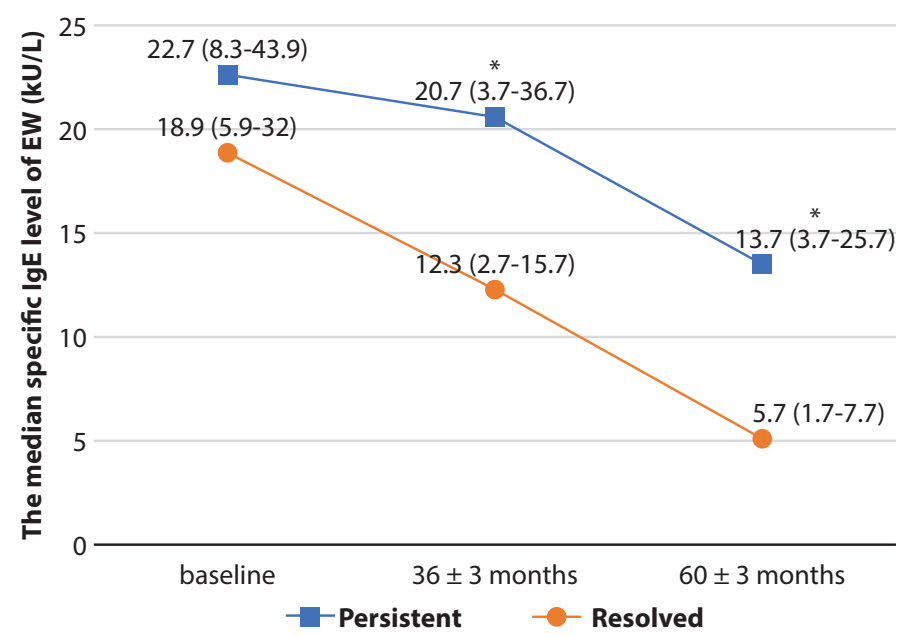

Figure 2. The median egg white-specific immunoglobulin $\mathrm{E}$ (IgE) levels in the resolved and persistent groups at 36 and 60 months of age $\left({ }^{\star} p=0.015,0.010\right.$, respectively)

EW, egg white 
(a)
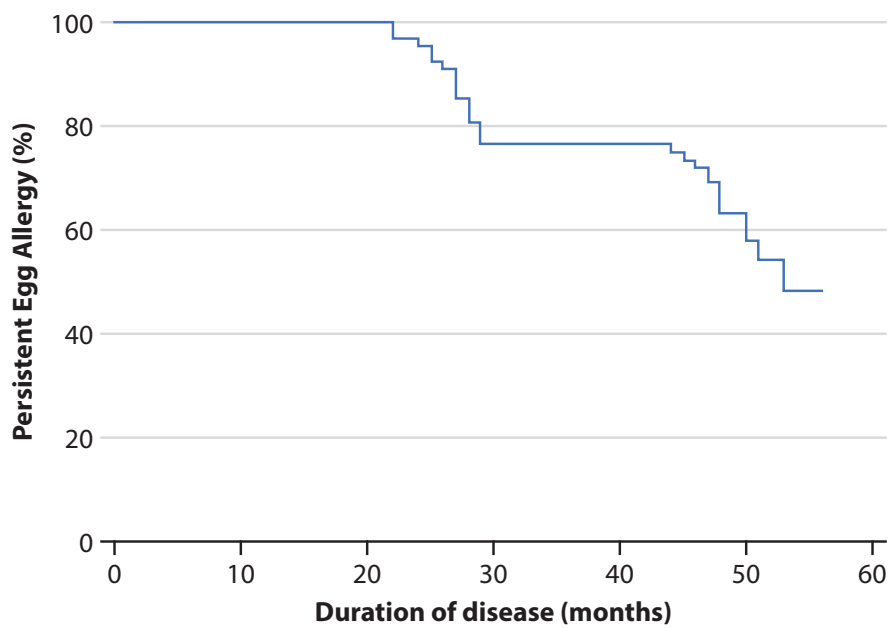

non AD ---- mild AD (b)
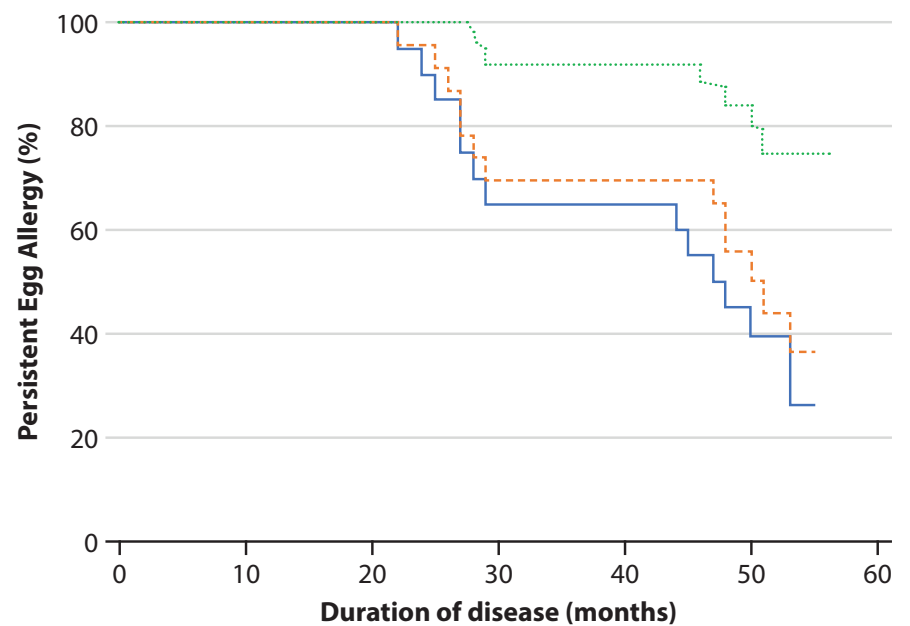

moderate to severe $A D$

Figure 3. Kaplan-Meier analysis of egg allergy remission over time. (a) Remission rate of 68 patients (b) Remission rates of groups according to atopic dermatitis severity $(P=0.008)$

However, the median [IQR] egg white-sIgE levels at $36 \pm 3$ and $60 \pm 3$ months were 10.5 [2.0-12.8] and 7.9 [1.2-10.8] $\mathrm{kU} / \mathrm{L}$ in the no $\mathrm{AD}, 13.8$ [2.7-17.7] and 9.1 [1.9-16.9] $\mathrm{kU} / \mathrm{L}$ in the mild $\mathrm{AD}$, and 22.5 [9.0-40.1] and 16.8 [9.3-30.8] $\mathrm{kU} / \mathrm{L}$ in the moderate to severe $\mathrm{AD}$ groups, respectively. The median egg white-sIgE levels at $36 \pm 3$ and $60 \pm 3$ months were higher in the moderate to severe AD group than in the other groups $(p=0.010)$ (Figure 4$)$.

The prognostic factors for egg allergy resolution in the univariate and multivariate Cox regression analyses are shown in Table 2. Baseline moderate to severe AD, high baseline egg white-sIgE ( $\geq 20 \mathrm{kU} / \mathrm{L}$ ), and HDM sensitization were significantly associated with persistent egg allergy in univariable analysis ( $p=0.010,0.031$, and 0.042 , respectively). However, only moderate to severe $\mathrm{AD}$ was a significant prognostic factor of persistent egg allergy in multivariable analysis (hazard ratio $=2.521,95 \%$ confidence interval $[\mathrm{CI}]$ : $1.855-3.211, p=0.012$ ). Sex, age at diagnosis, vaginal delivery, exclusive breastfeeding, concomitant allergic disease, family history of allergic diseases, anaphylaxis, HDM sensitization, and laboratory findings were not statistically related to persistent egg allergy.

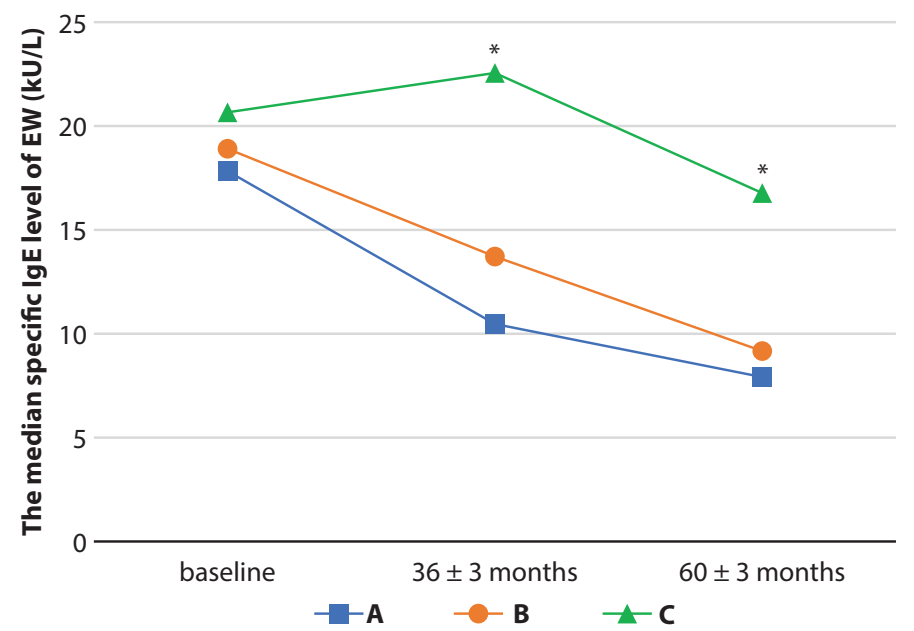

Figure 4. The median levels of specific immunoglobulin E (sIgE) to egg white

A: Egg allergy without atopic dermatitis

B: Egg allergy with mild atopic dermatitis

C: Egg allergy with moderate to severe atopic dermatitis ${ }^{*} p=0.01$

Table 2. Prediction of egg allergy resolution according to univariable and multivariable Cox analyses.

\begin{tabular}{|c|c|c|c|c|c|c|}
\hline \multirow{2}{*}{ Variables } & \multicolumn{3}{|c|}{ Univariable analysis } & \multicolumn{3}{|c|}{ Multivariable analysis } \\
\hline & Hazard ratio* & $95 \% \mathrm{CI}$ & p-value & Hazard ratio* & $95 \% \mathrm{CI}$ & p-value \\
\hline \multicolumn{7}{|l|}{ Sex } \\
\hline Male & 1 & & & & & \\
\hline Female & 0.945 & $(0.612-1.325)$ & 0.797 & & & \\
\hline \multicolumn{7}{|l|}{ Age at diagnosis (years) } \\
\hline$<1$ & 1 & & & & & \\
\hline$\geq 1$ & 0.996 & $(0.762-1.292)$ & 0.733 & & & \\
\hline
\end{tabular}


Table 2. (Continued)

\begin{tabular}{|c|c|c|c|c|c|c|}
\hline \multirow{2}{*}{ Variables } & \multicolumn{3}{|c|}{ Univariable analysis } & \multicolumn{3}{|c|}{ Multivariable analysis } \\
\hline & Hazard ratio* & $95 \% \mathrm{CI}$ & p-value & Hazard ratio* & $95 \% \mathrm{CI}$ & p-value \\
\hline \multicolumn{7}{|l|}{ Vaginal delivery } \\
\hline No & 1 & & & & & \\
\hline Yes & 0.912 & $(0.779-1.263)$ & 0.881 & & & \\
\hline \multicolumn{7}{|l|}{ Exclusive breastfeeding ${ }^{*}$} \\
\hline No & 1 & & & & & \\
\hline Yes & 0.927 & $(0.821-1.291)$ & 0.679 & & & \\
\hline \multicolumn{7}{|c|}{ Concomitant allergic disease } \\
\hline No & 1 & & & & & \\
\hline Yes & 1.231 & $(0.879-1.563)$ & 0.381 & & & \\
\hline \multicolumn{7}{|c|}{ Family history of allergic disease } \\
\hline No & 1 & & & & & \\
\hline Yes & 1.068 & $(0.770-1.479)$ & 0.693 & & & \\
\hline \multicolumn{7}{|l|}{ Anaphylaxis } \\
\hline No & 1 & & & 1 & & \\
\hline Yes & 1.383 & $(0.861-1.722)$ & 0.180 & 1.393 & $(0.837-2.320)$ & 0.202 \\
\hline \multicolumn{7}{|l|}{ Baseline AD severity } \\
\hline No & 1 & & & 1 & & \\
\hline Mild & 1.321 & $(0.961-1.613)$ & 0.098 & 1.287 & $(0.909-1.498)$ & 0.062 \\
\hline Moderate to severe & 2.768 & $(1.869-3.522)$ & 0.010 & 2.521 & $(1.855-3.211)$ & 0.012 \\
\hline \multicolumn{7}{|c|}{ Baseline EW-specific IgE (kU/L) } \\
\hline$<20$ & 1 & & & 1 & & \\
\hline$\geq 20$ & 1.345 & $(1.052-1.774)$ & 0.031 & 1.211 & $(0.892-1.447)$ & 0.071 \\
\hline \multicolumn{7}{|l|}{ HDM sensitization } \\
\hline No & 1 & & & 1 & & \\
\hline Yes & 1.346 & $(1.074-2.63)$ & 0.042 & 1.232 & $(0.891-1.932)$ & 0.174 \\
\hline \multicolumn{7}{|c|}{ Total IgE at diagnosis (kU/L) } \\
\hline$<200$ & 1 & & & & & \\
\hline$\geq 200$ & 1.093 & $(0.791-1.511)$ & 0.590 & & & \\
\hline \multicolumn{7}{|c|}{ Eosinophils at diagnosis (\%) } \\
\hline$<4$ & 1 & & & & & \\
\hline$\geq 4$ & 1.059 & $(0.756-1.486)$ & 0.737 & & & \\
\hline
\end{tabular}

Abbreviations: AD: atopic dermatitis., EW: egg white, HDM: house dust mite

${ }^{\star}$ Hazard ratio $>1$ indicates a proportional increase in the chance of persistent egg allergy 


\section{Discussion}

This retrospective cohort study based on OFC results investigated the natural history of immediate-type egg white allergy in Korean children. We found remission rates of $23.5 \%$ by 3 years of age and $47.1 \%$ by 5 years of age in the study subjects. The results of the Cox proportional regression model revealed that moderate to severe $\mathrm{AD}$ was the only significant predictor of poor egg allergy resolution.

The natural course of egg allergy is not yet fully understood. Most egg allergy develops in early infancy and resolves in most patients by school age. ${ }^{14}$ However, previous studies have shown that the reported tolerance rates $(11-81.5 \%$ by 3 years and $26-73 \%$ by 6 years) are highly variable. $^{14-20}$ In our study, $23.5 \%$ of the children developed tolerance towards eggs by 3 years of age and $47.1 \%$ by 5 years. This can be explained by differences in the present study population, such as race, concomitant atopic disease, and dietary habits as well as study methods.

The predictors for egg allergy resolution are also not yet well defined. Factors such as serum allergen-specific $\operatorname{IgE}$ level, skin prick test (SPT) wheal size, severity of previous reactions, eczema, age at diagnosis, and initial baked egg reactivity have been proposed. ${ }^{7,8,14,15}$ Several studies have examined the effect of $\mathrm{AD}$ on the resolution of food allergies in Asia. A Korean study reported that $90 \%$ of infants with egg allergy without $\mathrm{AD}$ had developed tolerance at 3 years of age, compared to $60 \%$ of patients with $\mathrm{AD} .{ }^{14}$ Recent Korean ${ }^{20}$ and Japanese ${ }^{21}$ retrospective studies have shown higher proportions of individuals with $\mathrm{AD}$ in the groups with persistent egg allergy compared to those in the tolerant groups. However, these studies did not evaluate AD severity in children with egg allergy.

The results of our study implied that egg allergy with moderate to severe $\mathrm{AD}$ was less likely to be resolved. The remission rates of egg allergy by 5 years of age in the no, mild, and moderate to severe $\mathrm{AD}$ groups were $65 \%$, $56.5 \%$, and $24 \%$, respectively. The group with moderate to severe $\mathrm{AD}$ had a significantly lower tolerance acquisition rate of egg allergy compared to those in the no AD and mild AD groups (Kaplan-Meier curve, $P=0.008$ ). The results of this study also showed higher median egg white-sIgE levels at 3 and 5 years of age in more severe $\mathrm{AD}$ compared to those for no and mild AD. Among participants with egg allergy and moderate to severe $\mathrm{AD}$, the median egg white-sIgE level had increased at 3 years $(22.5 \mathrm{kU} / \mathrm{L})$ of age compared to that at the time of diagnosis $(20.7 \mathrm{kU} / \mathrm{L})$. Epicutaneous sensitization through severely damaged skin may cause increased food-specific IgE levels, which could further delay food allergy resolution. This important finding suggests that active treatment of impaired skin barriers may expedite egg allergy resolution in patients with moderate to severe AD.

The baseline egg white-sIgE level is considered as a predictor of tolerance acquisition. ${ }^{14}$ Several studies have demonstrated a poor prognosis in patients with high egg white-sIgE levels. ${ }^{22-25}$ However, multivariable analysis in our study showed that high baseline egg white-sIgE level $(\geq 20$ $\mathrm{kU} / \mathrm{L}$ ) was not significantly related to persistent egg allergy.
A Korean study also reported that initial egg white-sIgE level was not statistically related to persistent egg allergy in multivariable analysis. ${ }^{26}$ Other studies have also shown an association between tolerance development and the rate of decrease in egg white-sIgE levels over time. ${ }^{720,27}$ The baseline egg white-sIgE level and the trend of reduced egg white-sIgE over time might be affected by the severity of skin barrier dysfunction in patients with egg allergy.

This study also showed significantly higher HDM sensitization in the persistent egg allergy group. While previous studies revealed reported that egg allergy or severe $\mathrm{AD}$ increased the risks of inhalant sensitization in late childhood, ${ }^{1,22,28,29} \mathrm{HDM}$ sensitization was not statistically related to persistent egg allergy in multivariable analysis in the present study. Further well-designed prospective large-population studies are needed to evaluate the correlation between food allergy resolution and inhalant allergen sensitization.

Our study had several limitations. First, this was a single-center study, and the patients may have had more severe allergies than those in general hospitals or clinics. Furthermore, this was a retrospective study and therefore might involve selection bias. The change in $\mathrm{AD}$ severity over time was also not evaluated. Nevertheless, the criteria for diagnosis and tolerance acquisition proposed in this study were applied more strictly to the medical record reviews. Patients who could not be administered egg whites because of their inherent antigen-specific IgE levels were excluded. In this investigation, $60(88.2 \%)$ patients underwent OFC, 8 $(11.8 \%)$ of whom had a definite history of egg allergy.

\section{Conclusion}

In conclusion, the results of this study showed a remission rate of $47.1 \%$ by 5 years of age and that moderate to severe $\mathrm{AD}$ may be a practical and important prognostic factor to predict persistent egg allergy.

\section{Conflicts of Interest}

The authors have no potential conflicts of interest to declare with respect to the authorship and/or publication of this article.

\section{Source of Funding with grant numbers None}

\section{Author Contributions}

- SHK, HYY, YHJ, YMK and HYK participated in the study design and conduct.

- SHK and HYK drafted the manuscript and performed the statistical analysis.

- All authors read and approved the final manuscript. 


\section{References}

1. Tham EH, Leung DYM. Mechanisms by Which Atopic Dermatitis Predisposes to Food Allergy and the Atopic March. Allergy Asthma Immun. 2019;11(1):4-15.

2. Saunes M, Oien T, Dotterud CK, Romundstad PR, Storro O, Holmen TL, et al. Early eczema and the risk of childhood asthma: a prospective, population-based study. Bmc Pediatr. $2012 ; 12: 168$.

3. Carlsten C, Dimich-Ward H, Ferguson A, Watson W, Rousseau R, DyBuncio A, et al. Atopic dermatitis in a high-risk cohort: natural history, associated allergic outcomes, and risk factors. Ann Allerg Asthma Im. 2013;110(1):24-8

4. Shaker M. New insights into the allergic march. Curr Opin Pediatr. 2014;26(4):516-20.

5. Silverberg JI. Comorbidities and the impact of atopic dermatitis. Ann Allergy Asthma Immunol [Preprint]. 2019 [cited 2019 Jul 10]. Available from: https://inkinghub.elsevier.com/retrieve/pii/S1081-1206 (19)30319-9

6. Bergmann MM, Caubet JC, Boguniewicz M, Eigenmann PA. Evaluation of food allergy in patients with atopic dermatitis. J Allergy Clin Immunol Pract. 2013;1(1):22-8

7. Sicherer SH, Wood RA, Vickery BP, Jones SM, Liu AH, Fleischer DM, et al. The natural history of egg allergy in an observational cohort. J Allergy Clin Immun. 2014;133(2):492-9.

8. Savage JH, Matsui EC, Skripak JM, Wood RA. The natural history of egg allergy. J Allergy Clin Immun. 2007;120(6):1413-7.

9. Song T.W. KKW, Kim WK, Kim JH, Kim HH, Park YM. Guidelines for the oral food challenges in children. Pediatr Allergy Respir Dis. 2012;22:4-20.

10. Yum HY, Yang HJ, Kim KW, Song TW, Kim WK, Kim JH, et al. Oral food challenges in children. Korean J Pediatr. 2011;54(1):6-10.

11. Nowak-Wegrzyn A, Assa'ad AH, Bahna SL, Bock SA, Sicherer SH, Teuber SS, et al. Work Group report: Oral food challenge testing. J Allergy Clin Immun. 2009;123(6):S365-83.

12. Bindslev-Jensen $\mathrm{C}$, Ballmer-Weber BK, Bengtsson $U$, Blanco $C$, Ebner C, Hourihane J, et al. Standardization of food challenges in patients with immediate reactions to foods - position paper from the European Academy of Allergology and Clinical Immunology. Allergy. 2004;59(7):690-7.

13. Hanifin JM, Rajka G. Diagnostic Features of Atopic-Dermatitis. Acta Derm-Venereol. 1980:44-7.

14. Kim JD, Kim SY, Kwak EJ, Sol IS, Kim MJ, Kim YH, et al. Reduction Rate of Specific IgE Level as a Predictor of Persistent Egg Allergy in Children. Allergy Asthma Immunol Res. 2019;11(4):498-507.

15. Savage J, Johns CB. Food Allergy Epidemiology and Natural History. Immunol Allergy Clin. 2015;35(1):45-59.
16. Gupta RS, Springston EE, Warrier MR, Smith B, Kumar R, Pongracic J, et al. The Prevalence, Severity, and Distribution of Childhood Food Allergy in the United States. Pediatrics. 2011;128(1):E9-17.

17. Gradman J, Mortz CG, Eller E, Bindslev-Jensen C. Relationship between specific IgE to egg components and natural history of egg allergy in Danish children. Pediat Allerg Imm-Uk. 2016;27(8):825-30.

18. Eggleston PA. Prospective studies in the natural history of food allergy. Ann Allergy. 1987;59(5 Pt 2):179-82.

19. Leonard SA, Sampson HA, Sicherer SH, Noone S, Moshier EL Godbold J, et al. Dietary baked egg accelerates resolution of egg allergy in children. J Allergy Clin Immunol. $2012 ; 130(2): 473-80 . e 1$.

20. Kim JH. Clinical and Laboratory Predictors of Egg Allergy Resolution in Children. Allergy Asthma Immunol Res. 2019;11(4):446-9.

21. Ohtani K, Sato S, Syukuya A, Asaumi T, Ogura K, Koike Y, et al Natural history of immediate-type hen's egg allergy in Japanese children. Allergol Int. 2016;65(2):153-7.

22. Boyano-Martinez T, Garcia-Ara C, Diaz-Pena JM, Martin-Esteban M. Prediction of tolerance on the basis of quantification of egg white-specific IgE antibodies in children with egg allergy. J Allergy Clin Immunol. 2002;110(2):304-9.

23. Xepapadaki P, Fiocchi A, Grabenhenrich L, Roberts G, Grimshaw KE Fiandor A, et al. Incidence and natural history of hen's egg allergy in the first 2 years of life-the EuroPrevall birth cohort study. Allergy. 2016;71(3):350-7

24. Rona RJ, Keil T, Summers C, Gislason D, Zuidmeer L, Sodergren E, et al The prevalence of food allergy: a meta-analysis. J Allergy Clin Immunol. 2007;120(3):638-46.

25. Jackson KD, Howie LD, Akinbami LJ. Trends in allergic conditions among children: United States, 1997-2011. NCHS Data Brief. 2013(121): $1-8$

26. Kim J, Chung Y, Han Y, Ahn K, Lee SI. The natural history and prognostic factors of egg allergy in Korean infants with atopic dermatitis Asian Pac J Allergy Immunol. 2009;27(2-3):107-14.

27. Shek LPC, Soderstrom L, Ahlstedt S, Beyer K, Sampson HA Determination of food specific IgE levels over time can predict the development of tolerance in cow's milk and hen's egg allergy. J Allergy Clin Immun. 2004;114(2):387-91

28. Ricci G, Patrizi A, Baldi E, Menna G, Tabanelli M, Masi M. Long-term follow-up of atopic dermatitis: retrospective analysis of related risk factors and association with concomitant allergic diseases. J Am Acad Dermatol. 2006;55(5):765-71.

29. Lowe AJ, Abramson MJ, Hosking CS, Carlin JB, Bennett CM, Dharmage SC, et al. The temporal sequence of allergic sensitization and onset of infantile eczema. Clin Exp Allergy. 2007;37(4):536-42. 